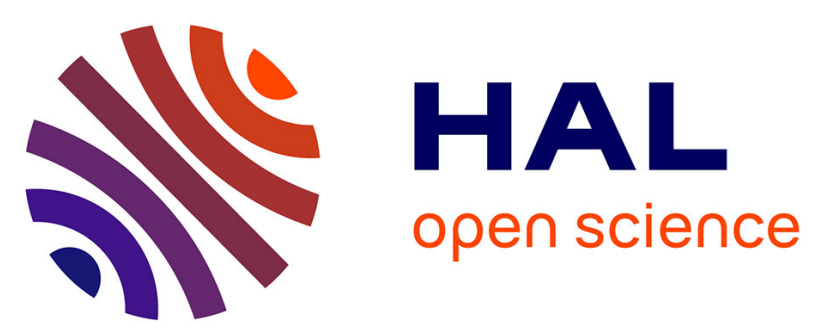

\title{
Binarization of the gray scale images of droplets during dropwise condensation on textured surfaces
}

\author{
Solmaz Boroomandi Barati, Martin Hélène, Jean-Charles Pinoli, Stéphane
}

Valette, Yann Gavet

\section{- To cite this version:}

Solmaz Boroomandi Barati, Martin Hélène, Jean-Charles Pinoli, Stéphane Valette, Yann Gavet. Binarization of the gray scale images of droplets during dropwise condensation on textured surfaces. QCAV 2019 - Quality Control by Artificial Vision, Institute IRIMAS de l' Université de Haute-Alsace (UHA), May 2019, Mulhouse, France. pp.111720R, 10.1117/12.2521447 . hal-02294810

\section{HAL Id: hal-02294810 \\ https://hal.science/hal-02294810}

Submitted on 23 Sep 2019

HAL is a multi-disciplinary open access archive for the deposit and dissemination of scientific research documents, whether they are published or not. The documents may come from teaching and research institutions in France or abroad, or from public or private research centers.
L'archive ouverte pluridisciplinaire HAL, est destinée au dépôt et à la diffusion de documents scientifiques de niveau recherche, publiés ou non, émanant des établissements d'enseignement et de recherche français ou étrangers, des laboratoires publics ou privés. 


\title{
Binarization of the gray scale images of droplets during dropwise condensation on textured surfaces
}

\author{
Solmaz Boroomandi Barati ${ }^{a}$, Hélène Martin ${ }^{b}$, Jean-Charles Pinoli ${ }^{b}$, Stéphane Valette ${ }^{c}$, Yann \\ Gavet $^{b}$, \\ ${ }^{a}$ Univ Lyon, Mines Saint-Etienne, LGF UMR CNRS 5307, SAINT-ETIENNE, France \\ ${ }^{b}$ Mines Saint-Etienne, LGF UMR CNRS 5307, SAINT-ETIENNE, France \\ ${ }^{c}$ Univ Lyon, Ecole Centrale de Lyon, LTDS UMR CNRS 5513, F-69134, LYON, France.
}

\begin{abstract}
In this research two methods for recognizing water droplets that are formed during dropwise condensation on the flat and pillared substrates are presented. The aim of these methods is to binarize the gray scale images of the droplets taken by a CCD camera in order to extract the information related to the droplets size and density.
\end{abstract}

Keywords: dropwise condensation, pillared substrates, image processing

\section{INTRODUCTION}

Dropwise condensation is the process during which water droplets form from a humid gas (or pure vapor) when its temperature goes below the dew point. ${ }^{1}$ This phenomenon can happen when the humid gas is in contact with a cold substrate with the temperature less than the dew point of the gas. In some applications the size and growth rate of these droplets on the cold substrate can be important because the smaller droplets show higher heat transfer rate. The growth rate of droplets can be controlled by applying different texturing patterns on the substrate. But sometimes due to the similarity between the texture shape and the droplets, the later are not recognizable. In this work, the image processing methods for recognizing the droplets on two substrates (flat and pillared) are presented. The aim of these methods is to binarize the images of the droplets in order to extract the information related to the droplets size and density.

Fig.1 shows the images of the droplets on the flat and pillared substrates. As it can be clearly seen the droplets on the flat substrates are recognizable, while on the pillared substrate the droplets are very similar to the pillars. Therefore, two different methods are used to binarize these images.

\section{METHODS}

\subsection{Image processing technique for the droplets on flat surface}

Due to the low contrast and inhomogeneity of small droplets, a preprocessing step is required. The Laplacian operator can be used in this regard, because it depends on the curvature instead of the gray level intensity and so it is robust with respect to contrast. The Laplacian filtering subtracts the pixel intensity of each droplets center point from the average of its neighborhood. A classical kernel $h_{L}$ is used to obtain the Laplacian by a convolution:

$$
h_{L}=\left[\begin{array}{ccc}
0 & 1 & 0 \\
1 & -4 & 1 \\
0 & 1 & 0
\end{array}\right]
$$

The Laplacian of an image highlights regions of rapid intensity change and is therefore often used for edge detection. The effect of Laplacian is illustrated in Fig.2. It can be seen in the filtered image that the contour of droplets is more visible and the gray level intensity of the small droplets is also improved with respect to the original image.

Further author information: (Send correspondence to Y.G..) Y.G.: E-mail: gavet@emse.fr 


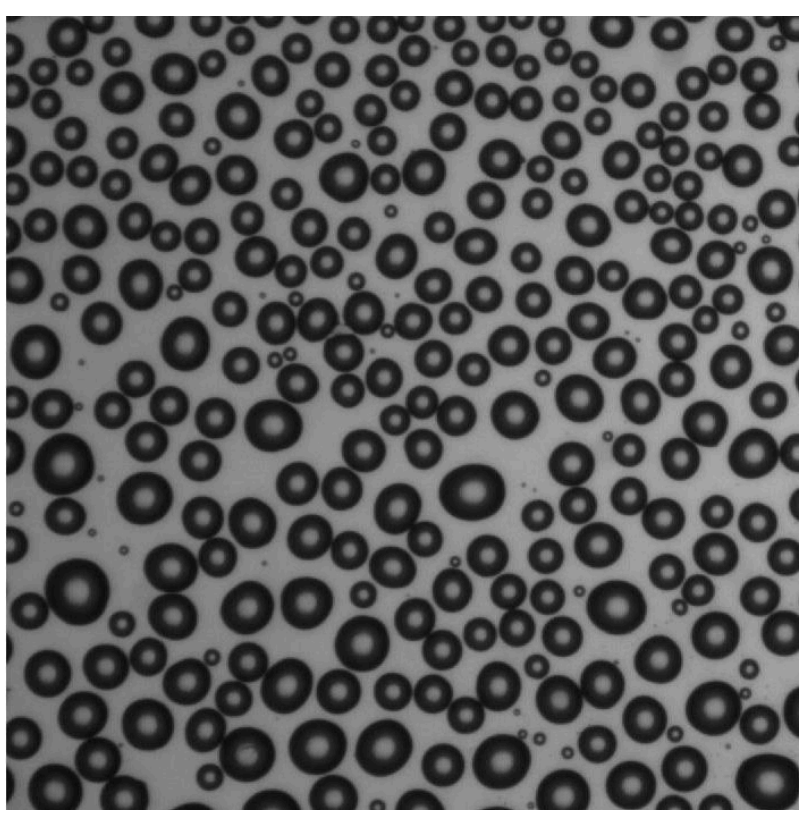

(a) Flat surface.

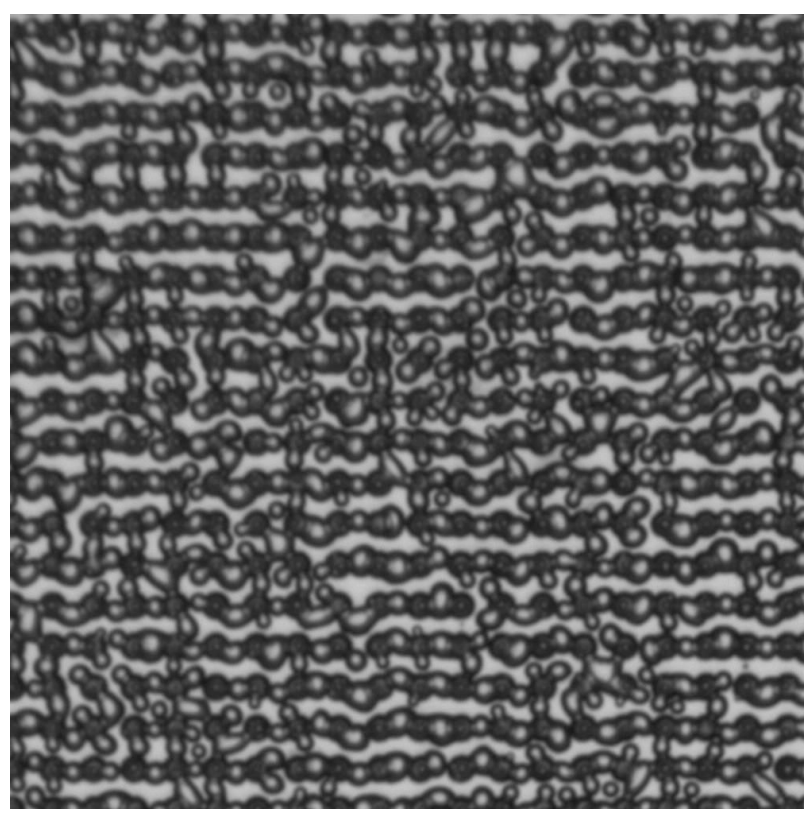

(b) Pillared surface.

Figure 1: The images of the droplets on two different substrates.

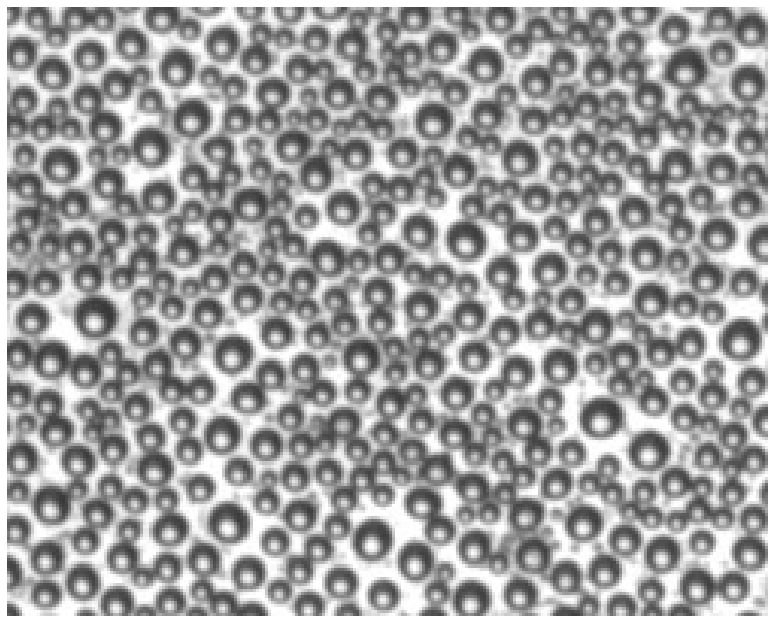

(a) Original image.

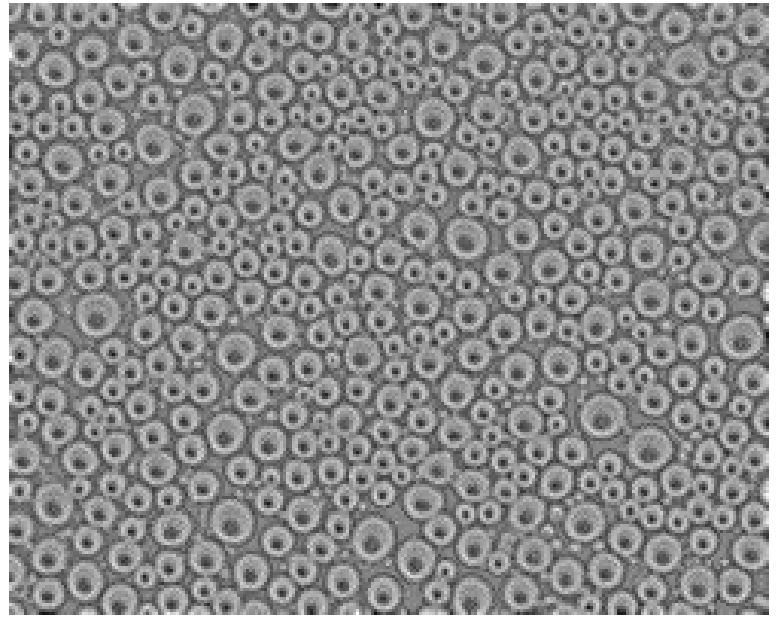

(b) Laplacian filter.

Figure 2: Illustration of the Laplacian filter, which is a first step in the segmentation process of the droplets. 
Then, the image is binarized via a local threshold technique, ${ }^{2,3}$ defined by Eq.1, where $t$ is the local threshold value (depending on the pixel coordinates $(x, y)$ ), $m$ is the local average value and $\sigma$ the local standard deviation. In this case, $k$ is a constant parameter of value 0.34 and $R$ is the dynamic range of the standard deviation (128 in our case).

$$
t(x, y)=m(x, y)\left[1+k\left(\frac{\sigma(x, y)}{R}-1\right)\right],
$$

The efficiency of this method can highly be improved by the use of integral image in order to compute $m$ and $\sigma$. The integral image is a linear algorithm that allows the computation of the mean of the values in a box of any dimension. The second step of this segmentation consists of the classical use of a distance map followed by a watershed, ${ }^{4,5}$ in order to perform the separation of contacting droplets (Fig.3).

\subsection{Image processing technique for the droplets on pillared surface}

In the case of pillared surfaces, the texturing patterns do not let the direct use of the technique presented in previous section, although they are adapted to different drop shapes and image conditions. Indeed, pillars have the same properties as drops, they have dark edges and bright centers. Moreover, when the drop shape becomes irregularly connected over the pillars, the previous method is not able to separate them and recognize each droplet.

The objective is to separate the droplets from the pillars in the serie of images acquired in a long period. Thus, at first steps, the droplets are really small and not visible, and the pillars can easily be detected. This first segmentation is used to suppress the pillars information in the next images.

As the droplets shape can vary a lot in the experiment, the algorithm should be adapted to the relative size of the droplet compared to the pillar size. Four categories are defined, very small, small, medium and large size droplets, as illustrated in Fig.4. These categories are defined using statistics of the grayscale intensities present in the image. Details can be found in: ${ }^{6} 4$ different algorithms are proposed, one for each category, based on:

- global threshold and pillars subtraction for very small droplets,

- Hough transform, pillars subtraction and watershed/distance map for small droplets,

- use of the convexity of the inner parts of the droplets in order to segment by watershed for medium droplets,

- and thresholding followed by mathematical morphology operators and watershed segmentation for the big droplets.

\section{RESULTS}

Fig.5 shows results of the segmentation of the droplets on flat surfaces at different times of the nucleation process. What is shown is that droplets have the same shapes and sizes at early times. After a while, coalescence (fusion) of droplets creates interstitial space between droplets, and the nucleation process starts again with small droplets. ${ }^{7}$

The validation of the methods is done by evaluating the $F$-measurement based on precision $P R$ and recall $R C$, defined by:

$$
F=\frac{2}{\frac{1}{P R}+\frac{1}{R C}}, P R=\frac{T P}{T P+F P}, R C=\frac{T P}{T P+F N}
$$

where $T P, F P, T N$ and $F N$ represent True Positive, False Positive, True Negative and False Negative, respectively. A high value of $P R$ indicates a missed droplets, while a high value of $R C$ indicates that algorithms detects non existing droplets. The groundtruth image segmentation is performed manually on a subsample of the image database.

The $F$-measure on flat surfaces is of $95 \%$ and of $54 \%$ on pillared surfaces. This is because the droplets on the flat surfaces are easier to recognize and so a classical algorithm works well for them. While on the pillared substrates, the droplets are very similar to the pillars and even calculating the convexity and the technique of watershed does not give a result precise enough for detecting them. 


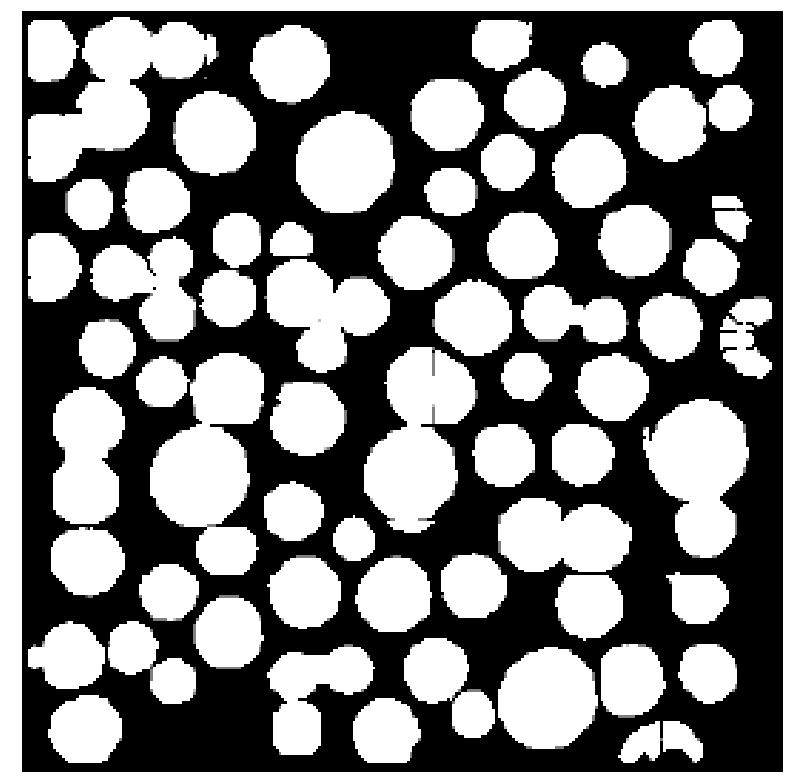

(a) Before reconstruction.

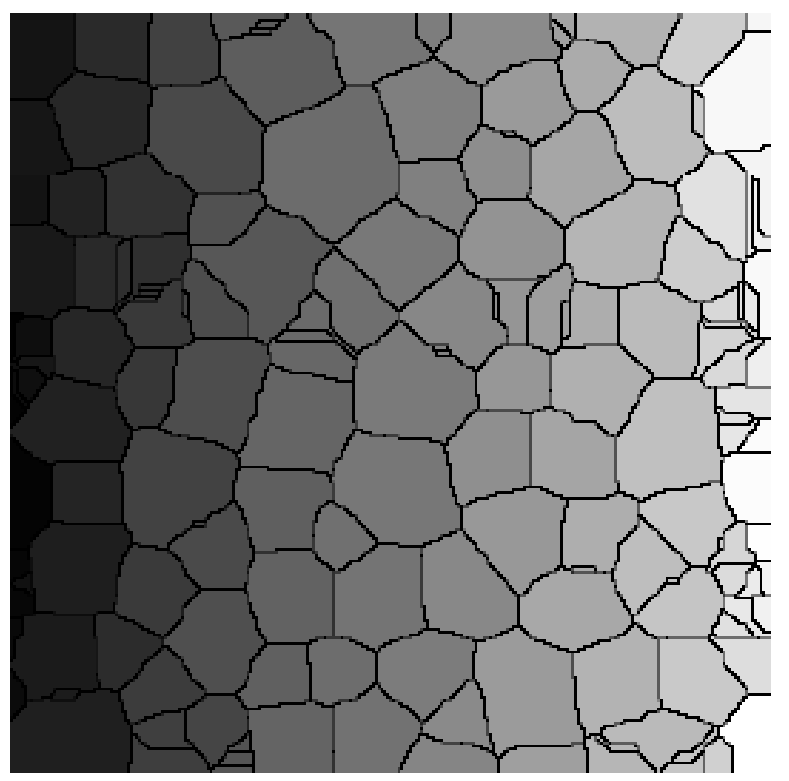

(c) Watershed segmentation.

Figure 3: Watershed following distance map are used to segment the image after the preprocessing step.

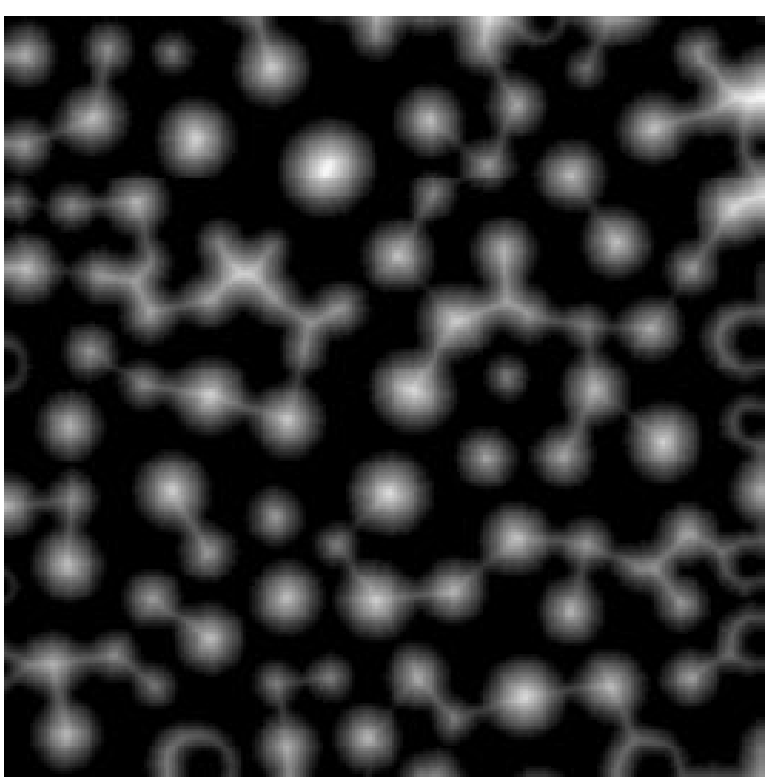

(b) Distance map operator.

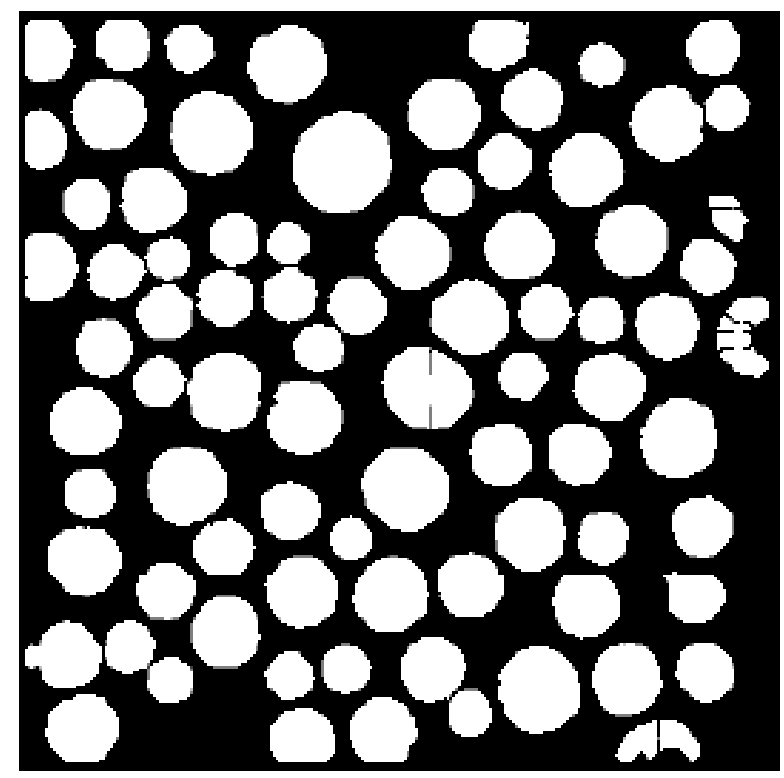

(d) Final binarized image. 


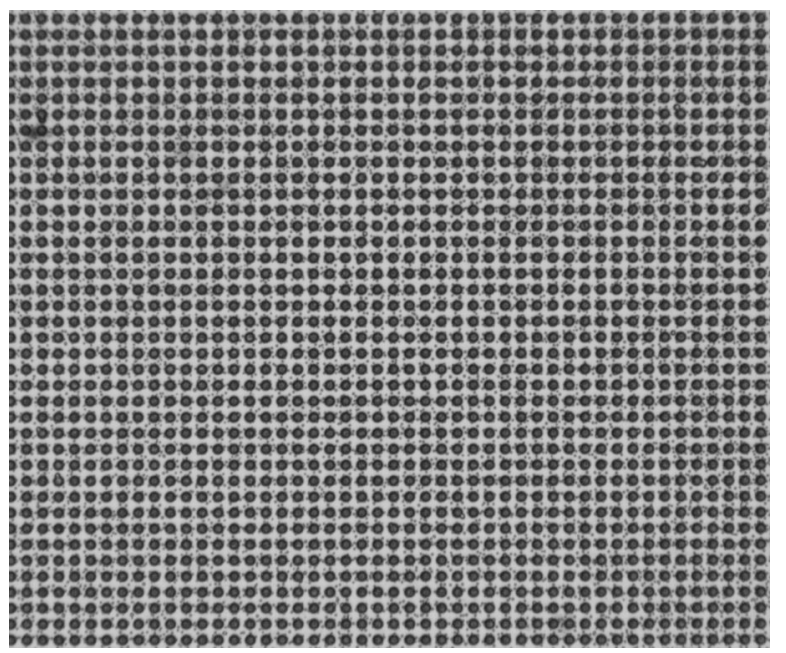

(a) Very small droplets.

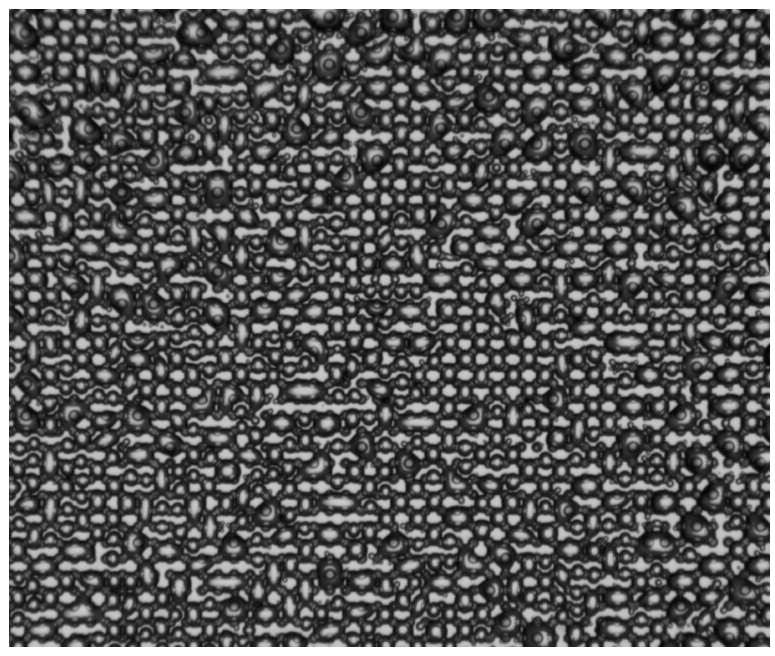

(c) Medium droplets.

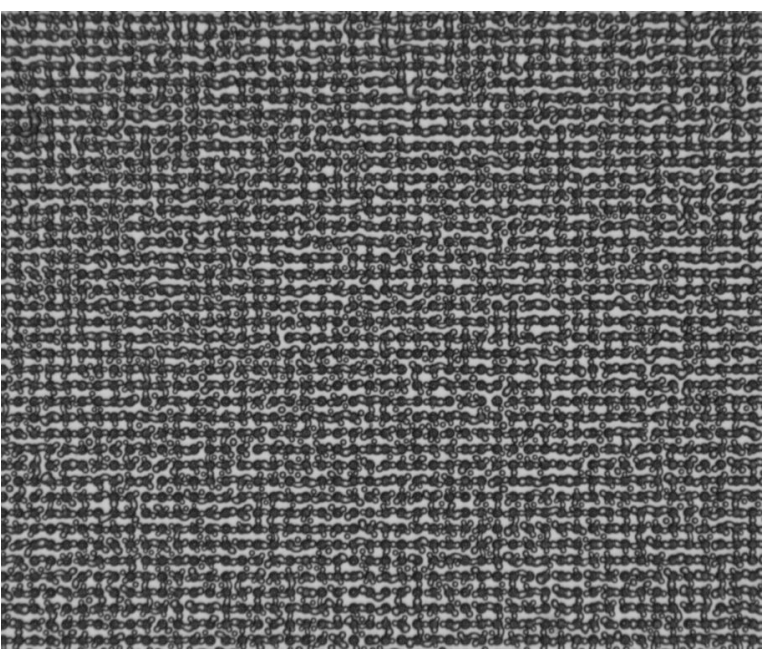

(b) Small droplets.

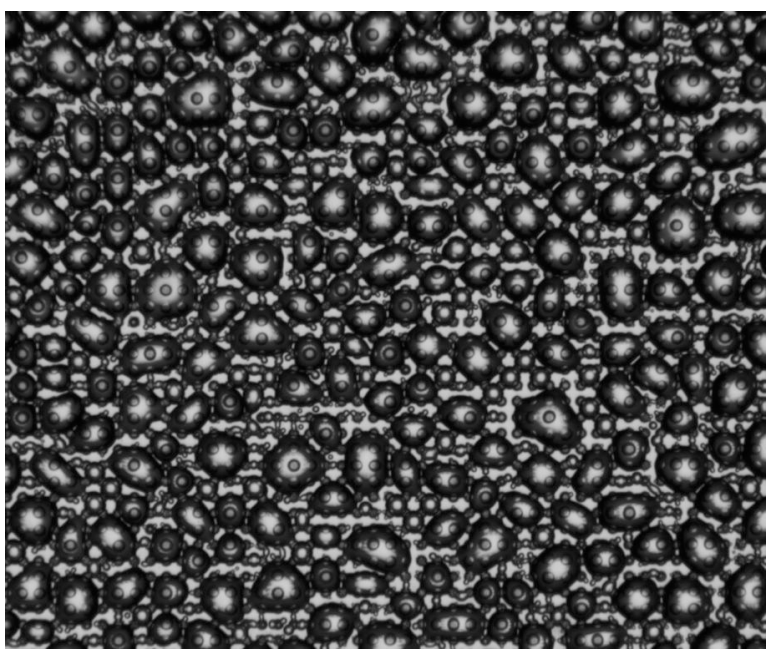

(d) Large droplets.

Figure 4: Categorization of the droplets by their size.
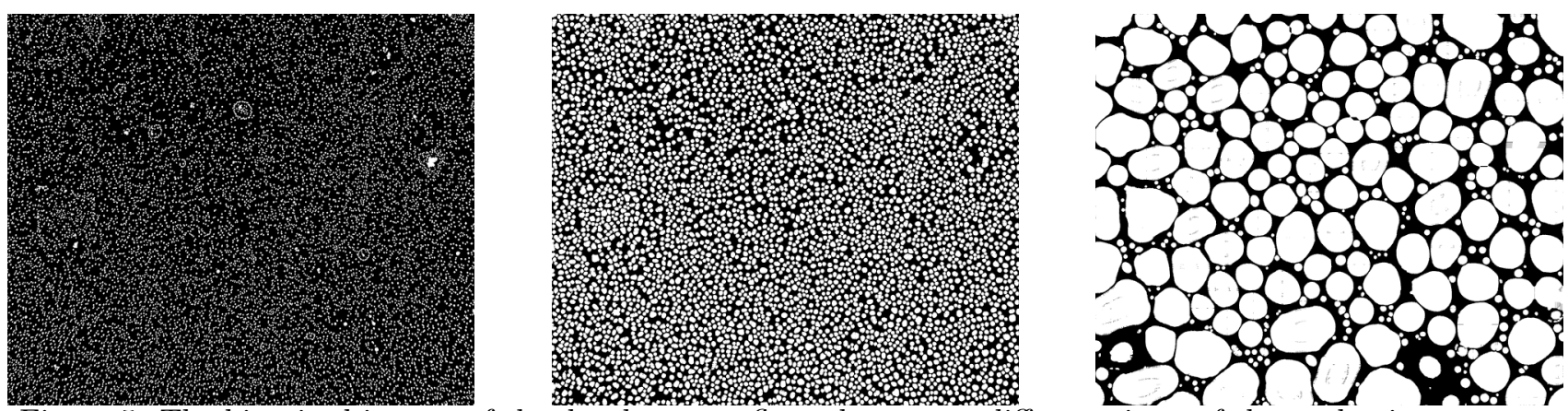

Figure 5: The binarized images of the droplets on a flat substrate at different times of the nucleation process. 

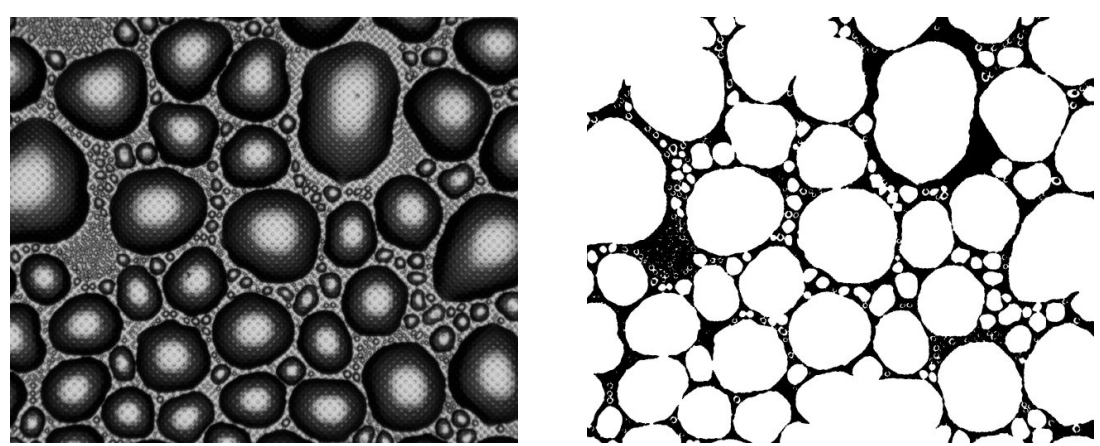

Figure 6: The binarized images of the droplets on a pillar substrate for big droplets. The presence of droplets of the size of the pillars complicates the segmentation of this type of image.

\section{CONCLUSION AND PERSPECTIVES}

This article presented grayscale images of droplets on flat and pillared surfaces. The segmentation of the images of these droplets has been performed in order to access information on sizes and shapes and quantify their evolution according to the time. On flat surfaces, the droplets are segmented with a high accuracy, because they are the only visible objects on the images. On pillared surfaces, the difficulty lays in the fact that the pillars have the same shape and sometimes also the same size as the droplets. Strategies based on a rough approximation of the sizes of the droplet, and a differentiation of the segmentation method according to them are proposed.

In other studies, ${ }^{8}$ we segmented and analysed images of the droplets condensation on topographically sinusoidal surfaces, in order to introduce more general surfaces like Gaussian random fields, that follow a Gaussian distribution in altitude and verify a spatial covariance. Our final objective is to study the spatial localisation of the droplets with regard to the surface (relative altitude, roughness, etc.).

\section{ACKNOWLEDGMENTS}

The authors would like to thank Nicolas Pionnier for the experimental results obtained during his PhD thesis funded by PSA Peugeot-Citroën in the context of OpenLab VATLyon between Peugeot-Citroën and LTDS laboratory. This work was funded by LABEX MANUTECH-SIZE (ANR-10-LBX-0075) within the programm "Investissement d'Avenir" (ANR-11-IDEX-0007) operated by the French National Research Agency (ANR).

\section{REFERENCES}

1. N. Kovalchuk, A. Trybala, and V. Starov, "Evaporation of sessile droplets," Current opinion in colloid and interface science 19(4), pp. 336-342, 2014.

2. J. Sauvola and M. Pietikäinen, "Adaptive document image binarization," Pattern recognition 33(2), pp. 225236, 2000.

3. F. Shafait, D. Keysers, and T. M. Breuel, "Efficient implementation of local adaptive thresholding techniques using integral images.," DRR 6815, p. 681510, 2008.

4. S. Beucher and C. Lantuejoul, "Use of watersheds in contour detection.," in International Workshop on image processing, real-time edge and motion detection/estimation, (Rennes, France), 1979.

5. P. Soille, Morphological image analysis: principles and applications, Springer Science \& Business Media, 2003.

6. H. Martin, S. B. Barati, J.-C. Pinoli, S. Valette, and Y. Gavet, "Segmentation of gray scale images of dropwise condensation on textured surfaces," World Academy of Science, Engineering and Technology, International Journal of Computer and Information Engineering 5(1), pp. 115-126, 2018.

7. S. B. Barati, N. Pionnier, J.-C. Pinoli, S. Valette, and Y. Gavet, "Investigation spatial distribution of droplets and the percentage of surface coverage during dropwise condensation," International Journal of Thermal Sciences 124, pp. 356 - 365, 2018.

8. S. B. Barati, Modeling and simulation of dropwise condensation on textured surfaces. PhD thesis, MINES Saint-Etienne, University of Lyon, 2018. 